\title{
A Novel and Modified Technique in the Prone Position for Radiotherapy Processes in Children
}

\author{
Ozkan Onal, ${ }^{1,}$ Emine Aslanlar, ${ }^{2}$ Cansu Ciftci, ${ }^{2}$ Merih Onal, ${ }^{3}$ and Jale Bengi Celik ${ }^{4}$ \\ ${ }^{1}$ Associate Professor, Selcuk University Medical Faculty, Department of Anesthesiology and Intensive Care, Konya, Turkey \\ ${ }^{2}$ M.D., Selcuk University Medical Faculty, Department of Anesthesiology and Intensive Care, Konya, Turkey \\ ${ }^{3}$ MD., Konya Educational and Training Hospital, Department of Otorhinolaryngology, Konya, Turkey \\ ${ }^{4}$ Professor, Selcuk University Medical Faculty, Department of Anesthesiology and Intensive Care, Konya, Turkey \\ "Corresponding author: Ozkan Onal, Selcuk University Medical Faculty, Department of Anesthesiology and Intensive Care, Konya, Turkey. E-mail: drozkanonal@selcuk.edu.tr
}

Received 2017 March 23; Accepted 2017 May 23.

\begin{abstract}
The i-gel has a thick airway tube and occasionally, achieving the airway can be difficult because of obstruction in the prone position. The authors aimed at solving this problem and used a modified i-gel airway in the prone position for radiotherapy processes in children.
\end{abstract}

\section{Dear Editor,}

The i-gel@ (Intersurgical Ltd., Wokingham, UK) is a single-use supraglottic airway device for use during general anesthesia. The main components of this device are a non-inflatable gel-like cuff that is claimed to achieve a perfect fit to the pharyngeal and laryngeal structures, and a thick airway tube with a gastric drain-tube orifice (1). The i-gel is often used during minor surgeries with the patient in the supine position. The i-gel has been used in the prone position by various authors in elective surgeries $(2,3)$. However, with its' thick airway tube, occasionally, achieving the airway can be difficult because of obstruction in the prone position. To solve this problem, the researchers of the current report use a modified i-gel airway tube.

For a 6-year-old patient with a brain tumor and metastasis to the spinal cord, 18 days of radiotherapy in the prone position was planned. For the procedure, which would last 25 minutes, radiotherapy was to be carried out under general anesthesia so that the patient would remain immobile. The patient was placed in the prone position, with his forehead and jaw on a hard plastic pillow. With this pillow, the distance between the floor and the patient's face was short, and could not be adjusted. When the i-gel was placed, a part of it that remained outside of the patient's mouth was long and pressed against the treatment table, thus, the patient could not be placed in the suitable position with complete accommodation of the jaw and forehead on the pillow. When a laryngeal mask airway (LMA) was placed instead of the i-gel, the LMA was pressed against the treatment table, preventing ventilation because it was folded. The problem could have been solved with a flexible LMA, yet, this was not available in a size suitable for a pediatric patient. Therefore, the medical team were forced to intubate the patient with a spiral number 5-mm internal diameter (ID) endotracheal tube (ETT). However, radiotherapy was expected to last for 18 days, and due to being in the prone position, the patient had to be intubated every day. In order to prevent trauma associated with daily intubation and extubation, the i-gel was modified to make it more flexible. The i-gel was cut to size 2 , just below the biting block and passed a number 5.5-mm ID spiral ETT from the inside (Figure 1). The cuff of the intubation tube was inflated with $10 \mathrm{~mL}$ of air and fixed the tube tightly within the i-gel. When the modified device was controlled, the i-gel and the ETT were not separated, although a marked force was exerted. On the second day of radiotherapy, with the patient under general anesthesia, the modified i-gel was used and the patient was moved to the desired position. The 25-minute treatment session was completed without any complications due to the spiral ETT and the flexible characteristics of the modified airway device. After the patient was placed in the supine position, the i-gel was removed by pulling it from the ETT.

In a previous study that was a source of inspiration for the current researchers, Sanuki et al. (4) used Magill forceps to remove a modified i-gel. They cut the i-gel to a length of $5.5 \mathrm{~cm}$, while the current researchers cut it to a length of $10 \mathrm{~cm}$, so that the i-gel was visible just below the teeth and could be manipulated and handled easily (Figure 2). In addition, while Sanuki et al. (4) inflated the cuff of the ETT with $2.5 \mathrm{~mL}$ of air, the current researchers did so with $10 \mathrm{~mL}$ of air and attached the tube firmly to the i-gel. They ensured that the tube was not separated from the igel, although a considerable amount of force was exerted. Due to these factors, the current researchers did not have 


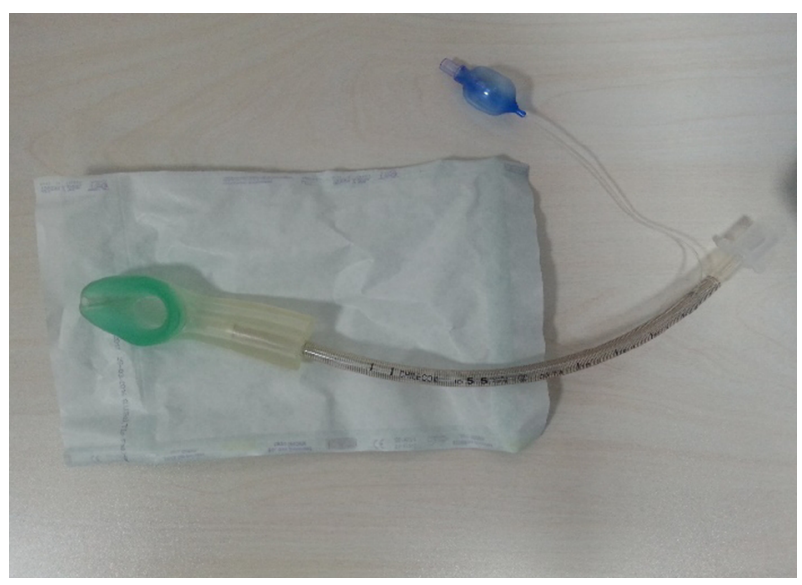

Figure 1. I-Gel

to use Magill forceps.

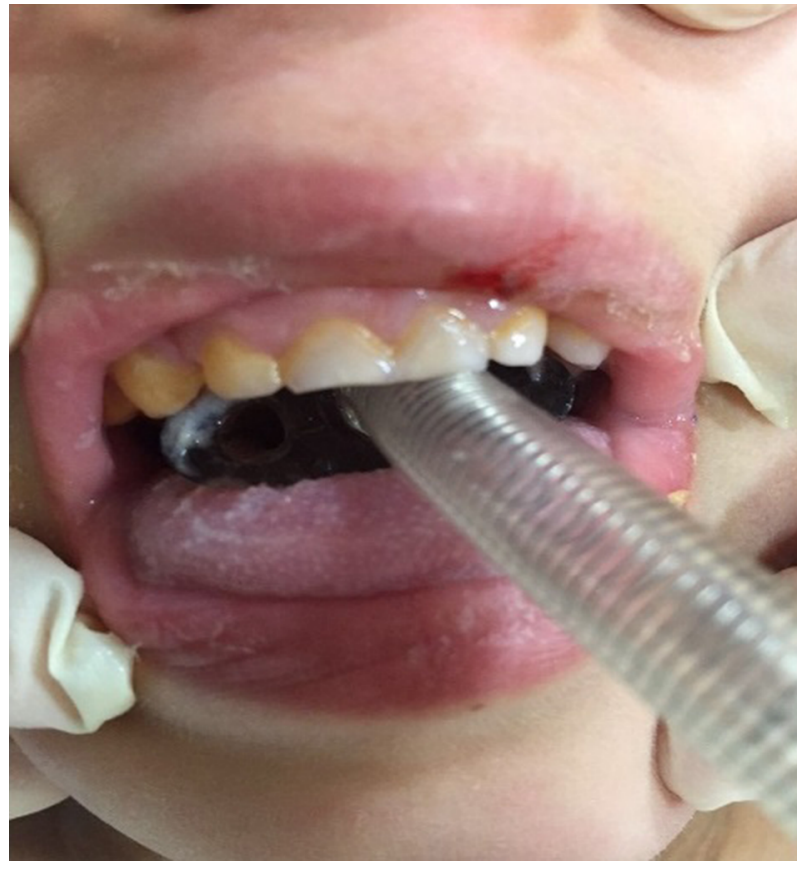

Figure 2. I-Gel
In conclusion, for short-term procedures that will be carried out with the patient in the prone position, unnecessary intubation may be avoided by means of a modified igel. In short procedures such as interventions for common tongue or lip injuries in children, by modifying the i-gel to the desired size, the surgeon's working area can be made more comfortable and airway safety can be secured without intubating the patient.

\section{Footnote}

Conflicts of Interest: The authors declare that they had no conflicts of interest.

\section{References}

1. Levitan RM, Kinkle WC. Initial anatomic investigations of the I-gel airway: a novel supraglottic airway without inflatable cuff. Anaesthesia. 2005;60(10):1022-6. doi:10.1111/j.1365-2044.2005.04258.x. [PubMed: 16179048].

2. Taxak S, Gopinath A, Saini S, Bansal T, Ahlawat MS, Bala M. A prospective study to evaluate and compare laryngeal mask airway ProSeal and igel airway in the prone position. Saudi J Anaesth. 2015;9(4):446-50. doi: 10.4103/1658-354X.159473. [PubMed: 26543466].

3. Taxak S, Gopinath A. Insertion of the i-gel airway in prone position. Min erva Anestesiol. 2010;76(5):381. [PubMed: 20395902].

4. Sanuki T, Sugioka S, Kotani J. Modified i-gel airway for oral surgery Anesth Prog. 2011;58(3):124-5. doi: 10.2344/11-00018.1. [PubMed: 21882988] 